\title{
A Palmprint Cryptosystem
}

\author{
Xiangqian $\mathrm{Wu}^{1}$, David Zhang ${ }^{2}$, and Kuanquan Wang ${ }^{1}$ \\ ${ }^{1}$ School of Computer Science and Technology, \\ Harbin Institute of Technology (HIT), Harbin 150001, China \\ $\{$ xqwu, wangkq\}@hit.edu.cn \\ http://biometrics.hit.edu.cn \\ ${ }^{2}$ Biometric Research Centre, Department of Computing, \\ Hong Kong Polytechnic University, Kowloon, Hong Kong \\ csdzhang@comp.polyu.edu.hk
}

\begin{abstract}
Traditional cryptosystems are based on passwords, which can be cracked (simple ones) or forgotten (complex ones). This paper proposes a novel cryptosystem based on palmprints. This system directly uses the palmprint as a key to encrypt/decrypt information. The information of a palmprint is so complex that it is very difficult, if not impossible, to crack the system while it need not remember anything to use the system. In the encrypting phase, a 1024 bits binary string is extracted from the palmprints using differential operations. Then the string is translated to a 128 bits encrypting key using a Hash function, and at the same time, an error-correct-code (ECC) is generated. Some general encryption algorithms use the 128 bits encrypting key to encrypt the secret information. In decrypting phase, the 1024 bits binary string extracted from the input palmprint is first corrected using the ECC. Then the corrected string is translated to a decrypting key using the same Hash function. Finally, the corresponding general decryption algorithms use decrypting key to decrypt the information. The experimental results show that the accuracy and security of this system can meet the requirement of most applications.
\end{abstract}

\section{Introduction}

Information security is becoming increasingly important in nowadays. Cryptology is one of the most effective ways to enhance the information security. In the traditional cryptosystems, information is encrypted using passwords. The simple passwords are easy to be memorized while they are also easy to be cracked. And the complex passwords are difficult to be cracked while they are also difficult to be remembered. In order to overcome this problem, some biometric feature-based encrypting/decrypting algorithms have been developed [1,2, 3, 4, 5, 6, 7,

The palmprint is a relatively new biometric feature [8, 9, 10, 11, 12, 13 and has several advantages compared with other currently available features 14: palmprints contain more information than fingerprint, so they are more distinctive; palmprint capture devices are much cheaper than iris devices; palmprints also contain additional distinctive features such as principal lines and wrinkles, 


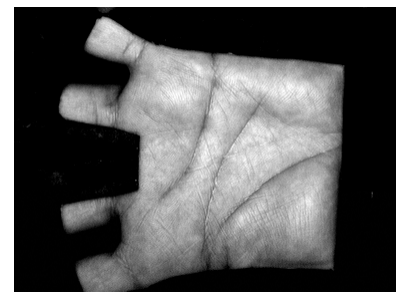

(a) Original Palmprint

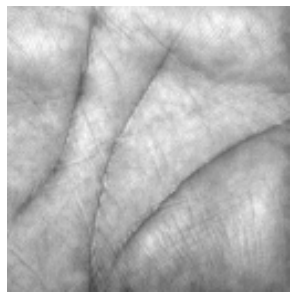

(b) Cropped Image

Fig. 1. An example of the palmprint and the normalized image

which can be extracted from low-resolution images; a highly accurate biometrics system can be built by combining all features of palms, such as palm geometry, ridge and valley features, and principal lines and wrinkles, etc. Therefore, it is suitable to use palmprints to implement a cryptosystem. Up to now, we failed to find any literature to discuss palmprint encryption. In this paper, we will use error-correcting theory to design a palmprint cryptosystem.

When palmprints are captured, the position and direction of a palm may vary so that even palmprints from the same palm may have a little rotation and translation. Furthermore, palms differ in size. Hence palmprint images should be orientated and normalized before feature extraction and matching. In this paper, we use the preprocessing technique described in [13] to align and normalize the palmprints. After preprocessing, the central part of the image, which is $128 \times 128$, is cropped to represent the whole palmprint. Fig. 11 shows a palmprint and the normalized image.

The rest of this paper is organized as follows. Section 2 describes the feature extraction and matching. Section 3 presents the palmprint cryptosystem. Section 4 contains some experimental results and analysis. And Section 5 provides some conclusions.

\section{Feature Extraction and Matching}

\subsection{DiffCode Extraction}

Let $I$ denote a palmprint image and $G_{\sigma}$ denote a 2D Gaussian filter with the variance $\sigma$. The palmprint is first filtered by $G_{\sigma}$ as below:

$$
I_{f}=I * G_{\sigma}
$$

where $*$ is the convolution operator.

Then the difference of $I_{f}$ in the horizontal direction is computed as following:

$$
\begin{aligned}
& D=I_{f} * b \\
& b=[-1,1]
\end{aligned}
$$

where $*$ is the convolution operator.

Finally, the palmprint is encoded according to the sign of each pixel of $D$ : 


$$
C(i, j)= \begin{cases}1, & \text { if } D(i, j)>0 \\ 0, & \text { otherwise }\end{cases}
$$

$C$ is called DiffCode of the palmprint $I$. The size of the preprocessed palmprint is $128 \times 128$. Extra experiments shows that the image with $32 \times 32$ is enough for the DiffCode extraction and matching. Therefore, before compute the DiffCode, we resize the image from $128 \times 128$ to $32 \times 32$. Hence the size of the DiffCode is $32 \times 32$. Fig. 2 shows some examples of DiffCode. From this figure, the DiffCode preserves the structure information of the lines on a palm.

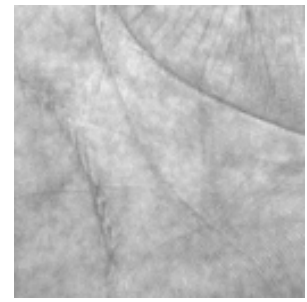

(a)

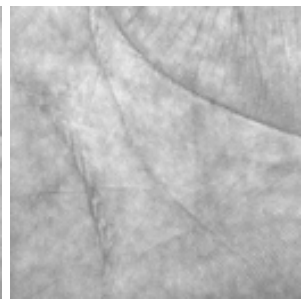

(b)

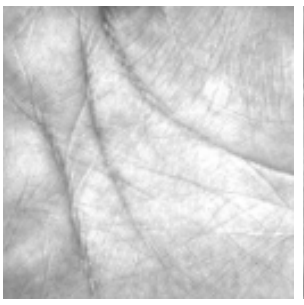

(c)

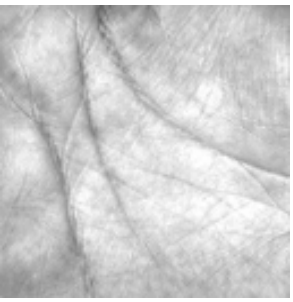

(d)

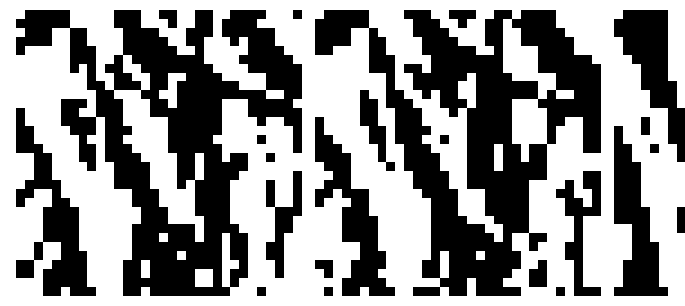

(e) (f)

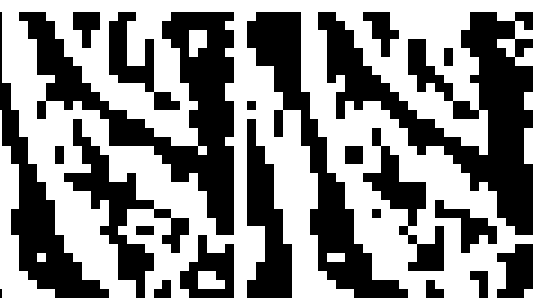

(g)

(h)

Fig. 2. Some examples of DiffCodes. (a) and (b) are two palmprint samples from a palm; (c) and (d) are two palmprint samples from another palm; (e)-(h) are the DiffCodes of (a)-(d), respectively.

\subsection{Similarity Measurement of DiffCode}

Because all DiffCodes have the same length, we can use Hamming distance to define their similarity. Let $C_{1}, C_{2}$ be two DiffCodes, their Hamming distance $\left(H\left(C_{1}, C_{2}\right)\right)$ is defined as the number of the places where the corresponding values of $C_{1}$ and $C_{2}$ are different. That is,

$$
H\left(C_{1}, C_{2}\right)=\sum_{i=1}^{32} \sum_{j=1}^{32} C_{1}(i, j) \otimes C_{2}(i, j)
$$

where $\otimes$ is the logical XOR operation. 
The matching distance of two DiffCodes $C_{1}$ and $C_{2}$ is defined as the normalized Hamming distance:

$$
D\left(C_{1}, C_{2}\right)=\frac{H\left(C_{1}, C_{2}\right)}{32 \times 32}
$$

Actually, $D\left(C_{1}, C_{2}\right)$ is the percentage of the places where $C_{1}$ and $C_{2}$ have different values. Obviously, $D\left(C_{1}, C_{2}\right)$ is between 0 and 1 and the smaller the matching distance, the greater the similarity between $C_{1}$ and $C_{2}$. The matching score of a perfect match is 0 . Because of imperfect preprocessing, there may still be a little translation between the palmprints captured from the same palm at different times. To overcome this problem, we vertically and horizontally translate $C_{1}$ a few points to get the translated $C_{1}^{T}$, and then, at each translated position, compute the matching distance between $C_{1}^{T}$ and $C_{2}$. Finally, the final matching distance is taken to be the minimum matching distance of all the translated positions.

\section{Palmprint Cryptosystem}

In general, the palmprints captured from the same hand at different time are not exactly same. However, they are similar enough to distinguish that they are from the same hand. That is, when the matching distance between the DiffCodes $C_{1}$ and $C_{2}$ is less than a threshold $T$, they should be regarded as being computed from the same hand, and $C_{2}$ should be able to decrypt the information which is encrypted using $C_{1}$. However, in general symmetric cryptosystems (eg.AES), it is impossible to successfully finish the decryption if the encrypting key and the decrypting key are not exactly same. To overcome this problem, we must transform $C_{2}$ to $C_{1}$ before using it for decryption. Since both $C_{1}$ and $C_{2}$ are binary strings with the same length, we can use the error-correct-coding theory to encode $C_{1}$ and get its error-correcting code, which can correct less than $T \times 1024$ errors, and then use this error-correcting code to correct $C_{2}$. If the matching distance between $C_{1}$ and $C_{2}$ is less than $T$, which means that $C_{1}$ and $C_{2}$ are from the same hand, $C_{2}$ can be exactly transformed to $C_{1}$ using the error-correcting code. And then the corrected $C_{2}$ can be used for decryption.

The principle of the palmprint cryptosystem is shown in Fig. 3 . In the encrypting phase, the $32 \times 32=1024$ bits DiffCode is extracted from the palmprints. Then the DiffCode is encoded to a fix length palmprint key (HC) using a Hash function (eg. MD5), and at the same time, an error-correct-code (ECC) of the DiffCode is generated using an existed algorithm (eg. BCH). Some general encryption algorithms (eg. AES) use this palmprint key to encrypt the secret information $S$. In decrypting phase, the 1024 bits DiffCode extracted from the input palmprint is first corrected using the ECC. Then the corrected string is encoded to a palmprint key (HC) using the same Hash function. Finally, the corresponding general decryption algorithms use this key to decrypt theinformation $(\mathrm{S})$.

To overcome the translation problem, we can get the $144 \times 144$ central part of the palmprint in the preprocessing of decryption phase, and then resize it to $36 \times 36$ to compute DiffCode. That is, in decryption phase, we get a DiffCode 


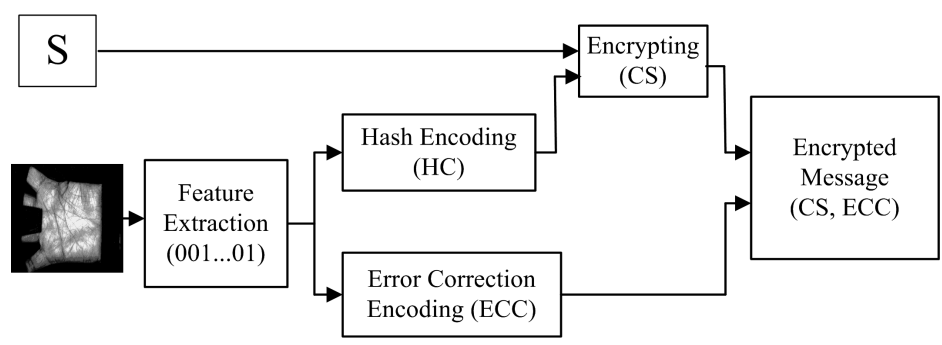

(a) Encrypting Phase

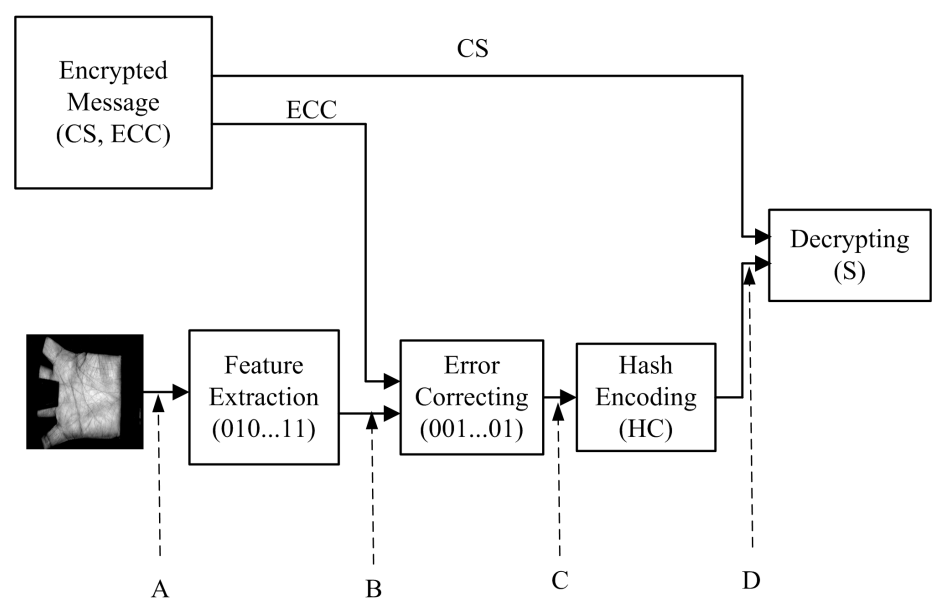

(b) Decrypting Phase

Fig. 3. Palmprint cryptosystem

with $36 \times 36$ size. From this larger DiffCode, we can get 25 DiffCodes with $32 \times 32$, which are used one by one for decryption until success. This process is equivalent to the translation the DiffCode vertically and horizontally from -2 to +2 points.

\section{Experimental Results and Analysis}

We employed the PolyU Palmprint Database [15] to test our system. This database contains 7,752 grayscale images captured from 386 different palms by a CCDbased device. These palmprints were taken from people of different ages and both sexes and were captured twice, at an interval of around two months, each time taking about 10 images from each palm. Therefore, This database contains about 20 images of each palm. The size of the images in the database is $384 \times 284$. In our 
experiments, all images were preprocessed using the preprocessing technique described in [13] and the central $128 \times 128$ part of the image was cropped to represent the whole palmprint. In the system, the Hash, error-correcting and encrypting algorithms are respectively selected as MD5, BCH and AES.

For a $(n, k, t) \mathrm{BCH}$ code, $n, k$ and $t$ respectively mean the length of the code, the length of the information and the number of the errors which can be corrected by this code.

For our system, $t$ can be computed using its distance threshold $T$ as following:

$$
t=1024 \times T \text {. }
$$

And $k$ should satisfy the following conditions:

$$
k \geqslant 1024
$$

If $k>1024$, we can append $(k-1024)$ zeros to the 1024 bits DiffCode to get the message with length $k$ and then encode it using $\mathrm{BCH}$ encoding.

Therefore, to error-correcting encoding, we should know the distance threshold of the system, which is dependent on the application. To investigate the relationship between the threshold and accuracy, each sample in the database is matched against the other palmprints in the same database. The matching between palmprints which were captured from the same palm is defined as a genuine matching. Otherwise, the matching is defined as an impostor matching. A total of $30,042,876(7,752 \times 7,751 / 2)$ matchings have been performed, in which 74, 086 matchings are genuine matchings. The FAR and FRR at different thresholds are plotted in Fig. 4. Some typical FARs, FRRs, the corresponding thresholds and the numbers of the error bits are listed in Table 1. We can select a threshold according to the requirement of the applications.

In our experiments, we choose the distance threshold as 0.2949. According to Table 1] the corresponding FAR, FRR and the number of errors which should be corrected are $0.0012 \%, 3.0169 \%$, and 302. According to the theory of $\mathrm{BCH}$ error-correcting-code, $(4095,1412,302) \mathrm{BCH}$ code can be used in our system. Now we analyze the attacks to this system.

If the attack happens at Point A (See Fig. 3), that is, the attacker uses some palmprints to attack the system. In this case, the possibility to successfully decrypt the message is about $0.0012 \% \approx 10^{-5}$, which means that to decrypt the message, a cracker has to find about $10^{5}$ different palmprints to try, which is very difficult to get so many palmprints in a short time.

If the attack happens at Point B (See Fig. 3), that is, the cracker attacks the system by directly generating the DiffCode for the error-correcting. The possibility to successfully decrypt the message in this way is $p$ :

$$
p=\frac{C_{1024}^{302}+C_{1024}^{301}+\cdots+C_{1024}^{1}+C_{1024}^{0}}{2^{1024}} \approx 2^{-134}
$$

If the attack happens at Point $\mathrm{C}$ (See Fig. 3), that is, the cracker generates the corrected DiffCode to attack the system, the possibility to success is $2^{-1024}$.

If the attack happens at Point D (See Fig. 3), that is, the cracker generates the hashed code to attack the system, the possibility to success is $2^{-128}$. 


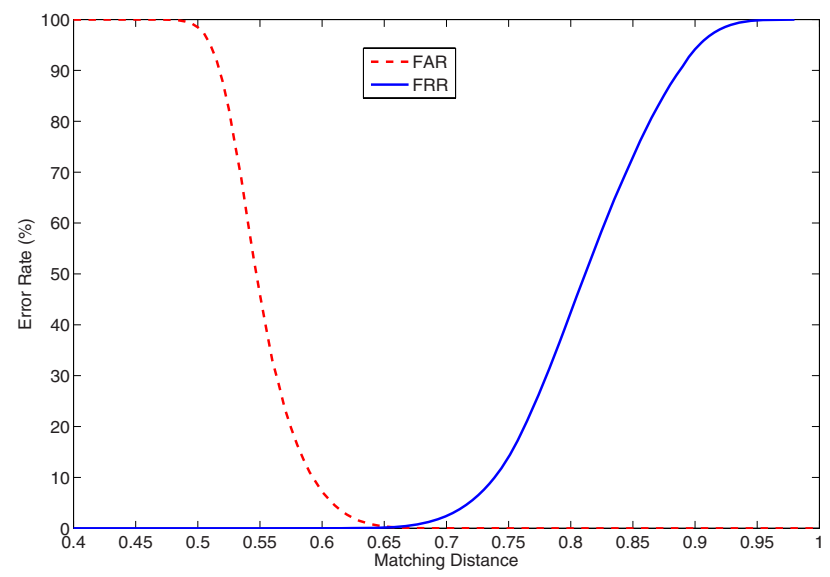

Fig. 4. The FAR and FRR at Different Threshold

Table 1. Typical FAR, FRR, corespoding thresholds and number of error bits

\begin{tabular}{|c|c|c|c|}
\hline Threshold & Number of Error Bits & FAR (\%) & FRR (\%) \\
\hline 0.3799 & 389 & 2.6862 & 0.0240 \\
\hline 0.3750 & 384 & 2.0133 & 0.0283 \\
\hline 0.3701 & 379 & 1.4784 & 0.0382 \\
\hline 0.3652 & 374 & 1.0976 & 0.0523 \\
\hline 0.3604 & 369 & 0.7799 & 0.0764 \\
\hline 0.3555 & 364 & 0.5458 & 0.1018 \\
\hline 0.3496 & 358 & 0.3786 & 0.1386 \\
\hline 0.3447 & 353 & 0.2558 & 0.2150 \\
\hline 0.3398 & 348 & 0.1663 & 0.2899 \\
\hline 0.3350 & 343 & 0.1092 & 0.3791 \\
\hline 0.3301 & 338 & 0.0663 & 0.4964 \\
\hline 0.3252 & 333 & 0.0399 & 0.6973 \\
\hline 0.3203 & 328 & 0.0238 & 0.9038 \\
\hline 0.3154 & 323 & 0.0135 & 1.2277 \\
\hline 0.3096 & 317 & 0.0075 & 1.5445 \\
\hline 0.3047 & 312 & 0.0042 & 1.9533 \\
\hline 0.2998 & 307 & 0.0022 & 2.4370 \\
\hline 0.2949 & 302 & 0.0012 & 3.0169 \\
\hline 0.2900 & 297 & 0.0006 & 3.6788 \\
\hline 0.2852 & 292 & 0.0003 & 4.4751 \\
\hline
\end{tabular}

\section{Conclusions}

This paper proposed a almprint cryptosystem. This system extracted binary DiffCode feature from palmprint and used the error-correcting theory to remove 
the difference between the DiffCodes from the same palms. The system can effectively encrypt and decrypt messages and it is almost impossible to crack it.

\section{Acknowledgements}

This work is partially supported by the National Natural Science Foundation of China (No. 60441005), the Key-Project of the 11th-Five-Year Plan of Educational Science of Hei Longjiang Province, China (No. HZG160), the Science and Technology Project of the Education Department of Hei Longjiang Province (No. 11523026) and the Development Program for Outstanding Young Teachers in Harbin Institute of Technology.

\section{References}

1. Uludag, U., Pankant, S., Prabhakar, S., Jain, A.K.: Biometric cryptosystems: issues and challenges. Proceedings of the IEEE 92, 948-960 (2004)

2. Freire-Santos, M., Fierrez-Aguilar, J., Ortega-Garcia, J.: Cryptographic key generation using handwritten signature. In: Proc. of SPIE, Biometric Technologies for Human Identificatin III (2006)

3. Uludag, U., Pankant, S., Jain, A.K.: Fuzzy vault for fingerprints. In: Kanade, T., Jain, A., Ratha, N.K. (eds.) AVBPA 2005. LNCS, vol. 3546, pp. 310-319. Springer, Heidelberg (2005)

4. Monrose, F., Reiter, M.K., Li, Q., Wetzel, S.: Using voice to generate cryptographic keys. In: A Speaker Odyssey, The Speaker Recognition Workshop, pp. 202-213 (2001)

5. Juels, A., Sudan, M.: A fuzzy vault scheme. In: Proc. IEEE International Symposium on Information Theory, IEEE Computer Society Press, Los Alamitos (2002)

6. Soutar, C., Roberge, D., Stojanov, S.A., Gilroy, R., Kumar, B.V.K.V.: Biometric encryption. ICSA Guide to Cryptography (1999)

7. Monrose, F., Reiter, M.K., Li, Q., Lopresti, D.P., Shih, C.: Towards speechgenerated cryptographic keys on resource constrained devices. In: Proc. 11th USENIX Security Symposium, pp. 283-296 (2002)

8. Zhang, D.: Palmprint Authentication. Kluwer Academic Publishers, Dordrecht (2004)

9. Wu, X., Zhang, D., Wang, K.: Palmprint Recognition. Scientific Publishers, China $(2006)$

10. Wu, X., Wang, K., Zhang, D.: Fisherpalms based palmprint recognition. Pattern Recognition Letters 24, 2829-2838 (2003)

11. Duta, N., Jain, A., Mardia, K.: Matching of palmprint. Pattern Recognition Letters 23, 477-485 (2001)

12. Han, C., Chen, H., Lin, C., Fan, K.: Personal authentication using palm-print features. Pattern Recognition 36, 371-381 (2003)

13. Zhang, D., Kong, W., You, J., Wong, M.: Online palmprint identification. IEEE Transactions on Pattern Analysis and Machine Intelligence 25, 1041-1050 (2003)

14. Jain, A., Ross, A., Prabhakar, S.: An introduction to biometric recognition. IEEE Transactions on Circuits and Systems for Video Technology 14, 4-20 (2004)

15. PolyU Palmprint Palmprint Database (http://www. comp.polyu.edu.hk/ biometrics/) 